\title{
SKED-controlled experimentation in an undergraduate instructional laboratory
}

\author{
F. E. BUTLER and MICHAEL G. GRISHAM \\ University of Iowa, Iowa City, Iowa 52242
}

\begin{abstract}
A SKED-based facility for undergraduate instructional research has been operating for over a year. The two-computer approach, with one machine for programming and the other for running experiments, is used. Criteria for computerized undergraduate research laboratories, benefits of SKED, and the two-computer approach are discussed.
\end{abstract}

The following criteria are proposed for control equipment in a laboratory facility to support undergraduate instruction: (1) Numerous classes and students will simultaneously use the equipment; timesharing should be provided. (2) Flexibility is essential. Various content areas require quite diverse paradigms. (3) Control of experimental procedures and collection of data is sufficient, since statistical analyses can generally be accomplished off-line. (4) Immediate availability of data facilitates instruction. (5) Control equipment should encourage increasingly individualized research. If each student can arrange his or her own experiments, original research can evolve from demonstrations of wellestablished phenomena. (6) Staff time is much more expensive in the long run than equipment; minimal instruction, supervision, and maintenance should be entailed. (7) Continuously accessible equipment allows students to fit laboratory time into their own schedules. (8) Control equipment must be compatible with existing subject interfaces, such as operant conditioning chambers or reaction time equipment. (9) Finally, minimal cost means wider applicability.

Most of the above criteria lead directly to consideration of computer systems. Timesharing, flexibility, minimal instruction, and minimal maintenance are characteristic of well-designed computer systems. In particular, such systems support increasingly original and complex research without requiring the acquisition of any instrumentation skills beyond the basic language used to program initial demonstration experiments. Wood, Sette, and Weiss (1975) recently reviewed minicomputer languages for psychological research. Software and hardware are readily available for SKED (Snapper, Stephens, \& Lee, 1974), ACT (Millenson, 1971), and SCAT (Polson, 1973). SKED is the least expensive,

Funds for the facility were provided by the Department of Psychology, University of Iowa, and by the Instructional Scientific Equipment Program, Division of Undergraduate Education in Science, National Science Foundation, Grant GY-10848, J. F. Knutson, Project Director. Reprints are available from M. G. Grisham, Department of Psychology, University of Iowa, lowa City, Iowa 52242. due to the relative simplicity of the required interface hardware (Wood et al., 1975).

A SKED system for paper-tape peripherals will simultaneously control 10 independent experiments. Each experiment may involve multiple subjects. Initiation and termination of an individual experiment do not affect other ongoing experiments. SKED applications have included operant conditioning (Snapper, Knapp, \& Kushner, 1970), recording and conditioning of bioelectrical potentials (Butler, 1975; Snapper, 1973; Butler, Note 1; Kadden, Note 2), autoshaping under classical conditioning procedures, and information processing research (Dingler, Uhlenhuth, \& Stern, 1975). Data may be labeled and are immediately available, an even more important consideration in educational research than in general (Restle \& Shaffer, 1974). SKED may also be used for exceedingly versatile simulation of models that specify a finite Markov chain (see Atkinson, Bower, \& Crothers, 1965, for examples of such models).

Using SKED involves preparation of a source program with the Digital Equipment Corporation (DEC) ${ }^{1}$ Symbolic Editor program. The SKED source must be converted into an object program by a compiler called COMP. Finally, a large program called RTS8PT will execute the experimental procedure and data collection when the SKED object is loaded. The Symbolic Editor, COMP, and RTS8PT programs must be loaded sequentially into the computer as they are needed, and the use of controls on the computer itself is required. This aspect of the SKED system is not optimal for educational applications where all access to the computer should be indirect, through a terminal or reader. Moreover, the inability to timeshare editing, compiling, and running restricts access to the system and imposes scheduling requirements.

One solution is a SKED system based on the DEC RTS/8 software. Although maximum benefit is extracted from a single machine, rather costly and complex peripheral equipment is entailed. An alternative is the two-computer approach suggested by Snapper (1974), with one machine devoted to editing and compiling programs and a second machine devoted to running 
experiments. We have adopted the two-computer solution and have combined the DEC Symbolic Editor and the SKED COMP compiler into a single program now available from the SKED Users Group. ${ }^{2}$ This combined program may be completely controlled from the Teletype associated with the programming system, while the independent run-time system conducts experiments.

The SKED source program is entered by Teletype into the programming system, with the usual Symbolic Editor commands available. A new command, X, produces first-pass compilation of the source. A second $X$ leads to second-pass compilation and output of the SKED object on the punch. Any error detected in compilation is indicated by COMP in the usual way, and control is transferred back to the Symbolic Editor automatically so that the error may be corrected. After error-free compilation is accomplished, a SKED source tape may be requested from the Symbolic Editor.

Repeated output of imperfect SKED sources is not required to determine if an error-free SKED program has been written. Therefore, considerable savings in time and supplies costs are realized. The SKED object and source should be punched successively on a single length paper tape. Then this tape may be readily identified by reading the source, and the source remains immediately available for later modification. The system supports program development for several SKED-based research laboratories, in addition to the undergraduate facility.

The existing undergraduate laboratory in the Department of Psychology at the University of Iowa consists of two DEC PDP-8/Ls, each with $12 \mathrm{~K}$ of memory and an ASR-33 Teletype. The programming system includes a high-speed punch for output of SKED sources and objects, and objects are entered into the runtime system by a high-speed reader. To preclude error, both the punch and reader are automatically activated whenever needed. The run-time system BUS-ALL digital interface (Butler, 1974), obtained from State Systems, ${ }^{3}$ contains a real-time clock, the reader interface, and 72 input and 72 output lines. Each line is wired to a patchboard, along with cables running to eight individual experimental rooms, to permit rapid allocation of particular inputs or outputs to different experiments. A 12-bit 16-channel A/D and 12-bit D/A are presently being installed.

The laboratory has controlled experiments involving response-produced brain stimulation, biological rhythms in activity, drug effects on operant baselines, and operant and classical conditioning procedures. All students initially used programs prepared for them, and some have progressed to writing their own programs for original studies. Instruction in SKED, provided by either a faculty member or graduate student teaching assistant, consisted of four $1 \mathrm{~h}$ meetings. During the first and second meetings, state notation diagrams were introduced and discussed. In the third and fourth meetings, these diagrams were translated into the SKED language and group exposure to operation of the programming and run-time systems was provided. The state notation diagram clearly facilitated acquisition of SKED. State notation diagrams appear side by side with SKED source programs throughout the undergraduate SKED manual currently being prepared in our department. ${ }^{4}$

In the 15 months that the instructional laboratory has been used, there have been some problems. SKED has a good set of error-detecting routines. However, the creativity and ingenuity of the students in finding ways to blow up the systems should not be underestimated. For example, one student could not get his new program to run the first time he loaded it, and when he reloaded it the run-time system crashed. It seems he wanted to collect the number of wheel turns in successive $15-\mathrm{sec}$ intervals, with a single data dump after $24 \mathrm{~h}$. Therefore, when the compiler requested number of counters, he had responded 5,760. Although this demand exceeded the maximum number of counters available to a single SKED program, the compiler failed to detect this error and produced a defective SKED object. Another undergraduate discovered a unique way to crash the programming system. He had lost his SKED source and believed he could obtain a new source by reading his SKED object into the programming system.

In general, two types of errors occur: errors that disrupt other ongoing experiments without system degradation and errors that degrade the system. Entering the wrong response or aborting the wrong station has no lasting effect on the system. However, typing CONTROL $C$ with a tape in the reader, typing shift $N$, dumping or clearing data in an inactive station, aborting a station not included in the current RTS8PT configuration, interacting with the computer switch register, and entering either an incorrect F3 starting address or incorrect number of parameters for an F3 all change the system software. Remedies for many of the above difficulties have been submitted to the SKED Users Group.

Without such error-detection routines available, approximately $5 \mathrm{~h}$ of staff time weekly have been required to keep the system software intact. As additional error detection was put into the software, less staff time was required. It is very helpful to routinely log all difficulties. A carbon copy of all Teletype activity would be valuable as well, because some students are reluctant to $\log$ their errors. Scheduling access to both the run-time system and available experimental rooms is required in any event, in addition to continuing consultation with individual students about programming.

The total cost of our two-computer facility was $\$ 12,200$; both PDP-8/Ls were purchased used and reconditioned. We have experienced no problems with any portion of the equipment except for failure of an integrated circuit in the BUS-ALL clock. A comparable laboratory using two PDP-8/As would be somewhat 
less costly and contain no used equiment. The $8 \mathrm{~K}$ of memory required for the programming system and the first $4 \mathrm{~K}$ field in the run-time system should be ferrite core, to avoid loss of the large Editor, COMP, and RTS8PT programs in the event of power failure. Additional memory in the run-time system, however, can be less expensive solid state RAM. Use of the PDP-8/A, which contains far fewer component cards than the $8 / \mathrm{L}$, would maximize the major virtue of the two-computer approach: If one machine fails, simple exchange of printed circuit cards between machines can isolate the problem card. Problems in a particular card would result in temporary shutdown of the programming system, awaiting return of the repaired card by DEC. Thus, minimal on-site technical support is required.

No more powerful or versatile control equipment is available at comparable cost. Both students and faculty have rapidly acquired the SKED language with minimal instruction. Numerous problems with the system have been discovered and eliminated. These problems have seldom occurred in SKED-based research laboratories, where there are fewer and more sophisticated users. At this point, it is apparent that the system meets the criteria proposed here for equipment to support undergraduate instructional research. Remarkable enthusiasm and commitment have ensued from providing a facility that encourages undergraduate students to develop and pursue their own research hypotheses.

\section{REFERENCE NOTES}

1. Butler, F. E. Use of SKED for classical and operant conditioning of single units in the rat's brain. Paper presented at the meeting of the American Psychological Association, Montreal, August 1973.

2. Kadden, R. M. Use of SKED computer system for online data recording of cardiac conditioning experiments. Paper presented at the meeting of the American Psychological Association, Montreal, August 1973.

\section{REFERENCES}

Atxinson, R. C., Bower, G. H., \& Crothers, E. J. An introduction to mathematical learning theory. New York: Wiley, 1965.

Butler, F. E. The BUS-ALL: An inexpensive set of interface for the SKED system. Behavior Research Methods \& Instrumentation, 1974, 6, 171-173.

Butler, F. E. Analog SKED. Behavior Research Methods \& Instrumentation, 1975, 7, 239-242.

Dingler, R. D., Uhlenhuth, E. H., \& Stern, M. SKED program for a modified Sternberg procedure. Behavior Research Methods \& Instrumentation, 1975, 7, 249-250.

MrLlenson, J. R. A programming language for on-line control of psychological experiments. Behavioral Science, $1971,16,248-256$.

Polson, P. G. SCAT: Design criteria and software. Behavior Research Methods \& Instrumentation, 1973, 5, 212-217.

Restle, F., \& Shaffer, W. O. Monitoring experimental results. Behavior Research Methods \& Instrumentation, $1974,6,262-266$.

SNAPPER, A. G. Use of notational system for digital control and recording. Behavior Research Methods \& Instrumentation, 1973, 5, 124-129.

SNAPPER, A. G. Alternative versions of SKED: Current systems and future plans. Behavior Research Methods \& Instrumentation, 1974, 6, 171-173.

SNapper, A. G., KNapp, J. A., \& Kushner, H. K. Mathematical descriptions of schedules of reinforcement. In W. N. Schoenfeld (Ed.), The theory of reinforcement schedules. New York: Appleton-Century-Crofts, 1970.

SNAPPER, A. G., Stephens, K. R., \& LeE, D. M. The SKED software system. Kalamazoo: SKED Users Group, 1974.

Wood, R. W., SETTE, W. F., \& Weiss, B. Interfacing the experimenter to the computer: Languages for psychologists. American Psychologist, 1975, 30, 230-238.

\section{NOTES}

1. DEC and PDP-8 are registered trademarks of Digital Equipment Corporation, Maynard, Massachusetts.

2. SKED Users Group, Department of Psychology, Western Michigan University, Kalamazoo, Michigan 49008.

3. State Systems, Inc., P.O. Box 2215, Kalamazoo, Michigan 49003.

4. Soon available from E. A. Wasserman, Department of Psychology, University of Iowa, lowa City, Iowa 52242. 